\title{
Teaching New Words to Children with Specific Language Impairment using Interactive Book Reading
}

\author{
Krista Voelmle, Speech-Language-Hearing: Sciences and Disorders, University of Kansas, \\ Lawrence, KS \\ Dr. Holly L. Storkel, Speech-Language-Hearing: Sciences and Disorders, University of \\ Kansas, Lawrence, KS \\ Disclosure: Krista Voelmle and Holly L. Storkel received salary support from the National \\ Institute on Deafness and Other Communication Disorders (DC012824) to support \\ completion of the research reported in this article.
}

Interactive book reading is an intervention strategy that consists of an adult reading a storybook to a child and deviating from the text to provide further information about the meaning, pronunciation, use, or spelling of a word that is likely new to the child (Wasid \& Bond, 2001). Interactive book reading is advantageous to young language learners because evidence shows that elaboration of words promotes better learning of new words than exposure without elaboration (Justice et al, 2005). Interactive book reading has moderate to large effects on word learning for typically developing children and children with low vocabulary associated with environmental challenges such as low socioeconomic status (see Marulis \& Neuman 2010 for review and metanalysis; Maynard, Pullen, \& Coyne 2010). In addition, it has been shown that children build their vocabulary in classroom settings through storybook reading (Elley, 1989). Thus, interactive book reading may be useful for speech-language pathologists (SLP) providing intervention in school setting. Our 
preliminary clinical trial provides evidence that children with SLI can learn new words through a high-intensity version of interactive book reading. The format of interactive book reading is flexible so that an SLP can easily administer the vocabulary words, supplementary information and read the story book to a group of children at one time or to a single child. This intervention also can be administered by other adults with the support and oversight of the SLP. For example, an SLP could train an SLP assistant or paraprofessional to administer the treatment, especially when using a scripted intervention like the one illustrated in this article. Furthermore it is possible for a parent or family member to be the intervention provider with coaching and support from the SLP (Crain-Thoreson \& Dale, 1999; Dale, Crain-Thoreson, Notari-Syverson \& Cole 1996; Pile et al. 2010). With adequate training, this would help to create more opportunities for practice outside of the therapy session, potentially leading to quicker word learning. If SLPs can provide an easy, brief and effective therapy tool for parents or other professionals to implement, this allows more opportunities for the child to work on building vocabulary in a therapy setting at school and at home.

\begin{abstract}
Although interactive book reading is an intervention strategy known to be effective in increasing vocabulary in typically developing children from higher and lower SES environments, there are few studies that have used this intervention with clinical populations that experience more severe language learning deficits. One population of particular interest is children with Specific Language Impairment (SLI). Children with SLI need 2-3 times as many exposures as typically developing children to learn new words
\end{abstract} (Gray, 2003; Rice, et al. 1994) so the traditional intervention approach to interactive book 
reading likely will not be sufficient to promote word learning in this population. Thus, a higher intensity version of interactive book reading is needed to support word learning by children with SLI.

\section{The Clinical Trial}

Our in-progress clinical trial, DC012824 Interactive Book Reading to Accelerate Word Learning by Children with SLI, is exploring the intensity of interactive book reading that children with SLI need to support word learning. Intensity in this case is defined as the number of exposures to each word. The four intensities being tested through this clinical trial include 12, 24, 36 and 48 exposures. Intensity 12 is the number of exposures that has been shown effective for children from low SES homes (Justice et al., 2005). Intensity 24 and 36 are 2 and 3 times more exposures, respectively. Thus, based on past studies of children with SLI, one of these intensities may be sufficient to promote word learning by children with SLI. Lastly, intensity 48 is 4 times as many exposures as the effective intensity in other studies of interactive book reading. This intensity is included to see whether children with SLI need even greater exposure when learning large numbers of words in naturalistic environments. Current results suggest that 36 exposures is the most promising intensity for children with SLI. Intensity 36 is illustrated as a guide to clinicians in creating a high intensity version of interactive book reading for children with language impairments. However, clinicians are cautioned to monitor each client's progress and adjust the intensity accordingly (see Komesidou \& Storkel, this issue, for guidance). 


\section{Interactive Book Reading Illustration: 36 Exposures}

\section{Stimuli}

The ongoing clinical trial uses the same stimuli as Justice, Meier, and Walpole's (2005) clinical trial of interactive book reading with Kindergarten children from low-income homes. The stimulus set consists of 10 commercially-available and Kindergartenappropriate books that contain "colorful illustrations that help narrate the story" for the children. These books contain the stimuli, which are "vocabulary words in the text that were unlikely to be known by the children" (Justice et al., 2005, p 21). For each book, six words were selected as potential treatment targets using common criteria (Beck et al., 2002). Specifically, the words were identified as "tier two" words, which are medium- to high-frequency words for mature language users and occur in multiple academic contexts. In addition, the words were likely to be unknown by kindergarteners. The stimuli for the project, displayed on the KU Word and Sound Learning Lab website shows the book titles and word selection for each book (https://wordlearning.drupal.ku.edu/appendix-mastertreatment-word-list). In our clinical trial, 5 books were assigned as treatment books and 5 books were assigned as untreated control books. Thus, each child was read 5 books in treatment and, through interactive book reading of those 5 books, was taught 30 words. Each word is elaborated or taught in four different ways: an explicit definition, the text in the book, a context sentence that uses the word and provides sufficient information to infer the word's meaning, and a synonym. Our website (https://wordlearning.drupal.ku.edu/appendix-master-treatment-word-list) shows each teaching context for each word. In creating teaching contexts, care was taken to select other 


\section{Individual Treatment Sessions}

During each individual treatment session the clinician read two books to the child: one during the first half of the session and one during the second half of the session. Each session lasted approximately 15-20 minutes. For each book, there were three parts to the treatment, which are illustrated in Figure 1: (1) Pre-Book Reading; (2) Book Reading; (3) Post-Book Reading. The three parts are a slight departure from some approaches to interactive book reading where all supplemental instruction occurs during book reading.
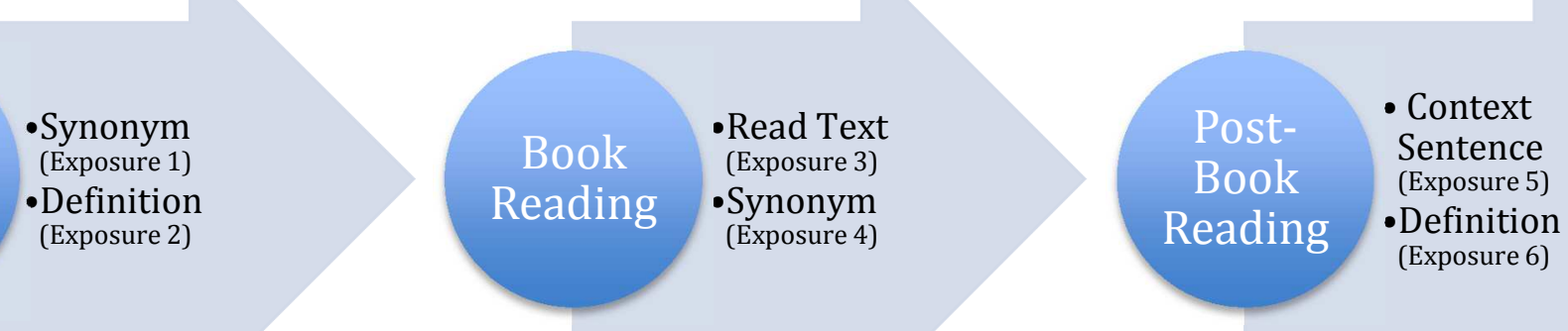

words that children with SLI were likely to know so that the elaboration would likely be helpful to the child with SLI. This particular interactive book reading treatment is constructed for children with SLI who exhibit vocabulary deficits and likely would have vocabulary goals on their IEP. However, traditional lower-intensity interactive book reading treatment can be utilized across children with milder vocabulary deficits. The methods for the interactive book reading treatment for children with SLI are illustrated for a treatment session. 
Given the high intensity of the treatment, it was hypothesized that the large number of exposures needed for children with SLI would interrupt the flow of the story, thereby hindering comprehension. Thus, the supplemental instruction was distributed before, during, and after book reading so that comprehension of the story would not be negatively impacted. A companion script was created for each book to ensure that the clinician delivered the planned level of exposure and support.

\section{Pre-Book Reading}

Before the book is read to the child the clinician talked about the words that the child would hear in the book. The six target words selected for that specific book were the focus of the pre-book reading activity. For example, when the target book was Otis (Bynum, 2000) the target words in that book (i.e., hauled, ripe, sidelines, hooves, silky, spotless) were previewed before the book was read. In this part of the treatment the clinician had a stack of six picture cards with each picture corresponding to one of the target words. Specifically, each picture was intended to illustrate the definition of the target word. The clinician showed the picture, provided the synonym and then provided the definition of the word. Below is an example for the target word "ruffle."

Show the picture card for "ruffle." 


\section{Storybook Reading}

This part of the treatment paralleled traditional interactive storybook reading in that the clinician departed from the text of the book to provide supplementary instruction as noted in the accompanying script. To help prompt the clinician to provide supplemental information, the target words were highlighted in the book with a box so that the clinician knew when to consult the script. The book text also was marked with an asterisk at the point in the text where the supplemental information from the script should be inserted. 
Generally, supplemental information was inserted at the end of a sentence or clause so that the flow of the story was not interrupted. An example for "ruffle" is shown below.

Book description: The target word "ruffle" occurs on page, 4 . The illustration has all the farm animals outside the barn in the field. The children are turning around in the car and waving goodbye to the animals as they drive away.

Clinician: "They had no rides to share, no tug-of-war to play, no one to scratch behind their ears or ruffle their feathers" (Bruss, 2001, p. 4). Ruffle is like disturb. (Synonym)

After the entire book has been read, post-book reading was initiated.

\author{
Post-Book Reading \\ Talking about the words that the child heard in the storybook was the last step of \\ treatment for each book during the treatment session. This part of the treatment was \\ similar in method to the pre-book reading. However, in this case the clinician discussed the \\ words in context sentences and repeated the definition. In addition, a different picture was \\ used, namely a picture that reflected the context sentence provided. The use of different \\ pictures added varied visual support for the children. Below is an example for "ruffle."
}

Show the picture card for "ruffle." 
Figure 3. Post-book reading stimulus card. Photo downloaded from http://duanerussell.com/Jordan/index.htm.

Clinician: Dad likes to ruffle my hair and make me laugh. (Context Sentence) Ruffle means to move something that is even to make it messy. (Definition)

This procedure was completed for each of the six target words in the book and then the second book was introduced following the same procedures. Once the treatment procedures were completed for the two books the treatment session ended.

Note that these treatment procedures result in the child hearing each word 6 times with accompanying visual support and information about the meaning of the word. Exposures 1 (Synonym) and 2 (Definition) were completed in the Pre-Book Reading, exposures 3 (Text 
in book) and 4 (Synonym) were provided in the Book Reading, and exposures 5 (Context sentence) and 6 (Definition) were delivered in the Post-Book reading. Also note that the teaching included the following repetitions of the teaching contexts: an explicit definition $(n=2)$, the text in the book $(n=1)$, a context sentence that used the word and provided sufficient information to infer the word's meaning $(n=1)$, and a synonym $(n=2)$.

\section{Repetition of Treatment Sessions}

To achieve 36 exposures to each word, treatment sessions for each book were repeated 6 times (i.e., 6 exposures per treatment session per book x 6 treatment sessions per book= 36 total exposures per book). This is illustrated in Figure 4. Note that books were rotated as they were repeated so that the order of the books did not stay consistent throughout treatment. This was done to guard against boredom or habituation. In the ongoing clinical trial, children were seen two times per week. Thus, as shown in Figure 4, the treatment lasted approximately 7.5 weeks, if no scheduled sessions were missed. 


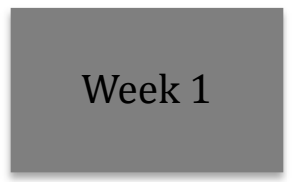

\section{Tx Session 1}

- Book 1 (1st )

-Book 2 (1st)

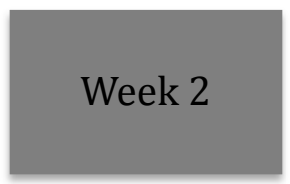

\section{Tx Session 3}

-Book 5 (1st)

-Book 1 (2nd)

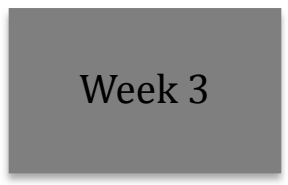

\section{Tx Session 5}

-Book 3 (2nd)

-Book 5 (2nd)

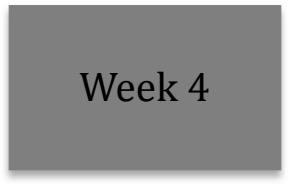

\section{Tx Session 7}

- Book 2 (3rd)

- Book 5 (3rd)

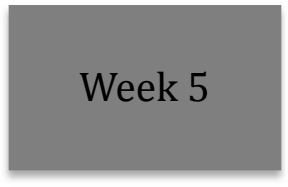

\section{Tx Session 9}

-Book 3 (4th)

-Book 2 (4th)

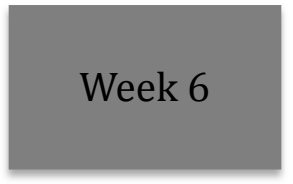

\section{Tx Session 11}

-Book 2 (5th)

-Book 1 (5th)

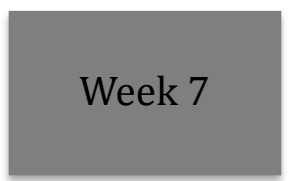

Tx Session 13

-Book 1 (6th)

-Book 5 (5th)
Tx Session 2

-Book 3 (1st)

-Book 4 (1st)

\section{Tx Session 4}

- Book 2 (2nd)

-Book 4 (2nd)

\section{Tx Session 6}

- Book 1 (3rd)

-Book 4 (3rd)

\section{Tx Session 8}

-Book 3 (3rd)

-Book 1 (4th)

\section{Tx Session 10}

-Book 5 (4th)

-Book 4 (4th)

Tx Session 12

-Book 4 (5th)

-Book 3 (5th)

\section{Tx Session 14}

-Book 4 (6th)

-Book 2 (6th)

\section{Tx Session 15 \\ Week 8 \\ -Book 5 (6th) \\ -Book 3 (6th)}

Figure 4 Visual Schedule for Intensity 36. Each book was read 6 times (see number in parentheses) and the order of the books was rotated across sessions.

\section{Can this Treatment be Used in Practice?}

Once the initial set-up of the treatment is completed (e.g., finding pictures, generating the script, etc.), interactive book reading treatment with an intense vocabulary training component is easy to carry out whether you are an SLP or another adult in the child's life. Due to the straightforwardness of the treatment, this type of approach can be utilized with 
children with SLI to build vocabulary in or outside of the children's regularly scheduled therapy sessions. Specifically, interactive book reading is a tool that can allow SLPs to support greater involvement of parents and caregivers through take-home work. That is, the SLP can provide copies of materials (e.g., a copy of the storybook or the picture cards) along with a script or game to guide home practice. Building vocabulary in children with SLI can lead to better language comprehension (Beck, Perfetti, \& McKeown, 1982), more efficient literacy skills (Roskos, et al., 2008) and overall improved academic achievement (Marulis \& Neuman, 2010). Interactive book reading is one approach that may prove successful in accelerating word learning by children with SLI. However, given the preliminary stage of our clinical trial, it is important that clinicians evaluate the success of this approach for specific clients. Komesidou and Storkel (this issue) illustrates learning profiles observed to date and provides guidance on adapting the treatment for children exhibiting different profiles during treatment. 


\section{References}

Beck, I. L., Perfetti, C. A., \& McKeown, M. G. (1982). Effects of long-term vocabulary instruction on lexical access and reading comprehension. Journal of Educational Psychology. 74(4). 506-521.

Beck, I. L., McKeown, M. G., \& Kucan, L. (2002). Bringing words to life. New York: Guilford Press.

Bruss, D. (2001). Book! Book! Book! New York: Arthur A. Levine Books.

Bynum, J. (2000). Otis. New York: Harcourt.

Cooper, H. (1993). The bear under the stairs. New York: Penguin Books.

Crain-Thoreson, C., \& Dale, P. S. (1999). Enhancing linguistic performance: Parents and teachers as book reading partners for children with language delays. Topics in Early Childhood Special Education, 19(1), 28-39.

Dale, P. S., Crain-Thoreson, C., Notari-Syverson, A., \& Cole, K. (1996). Parent-child book reading as an intervention technique for young children with language delays. Topics in Early Childhood Special Education, 16(2), 213-235.

Elley, W. B. (1989). Vocabulary acquisition from listening to stories. Reading Research Quarterly, 24, 174-187.

Gackenbach, D. (1977). Harry and the terrible whatzit. New York: Clarion Books.

Gray, S. (2003). Word-learning by preschoolers with specific language impairment: What predicts success? Journal of Speech, Language, and Hearing Research, 46(1), 56-67.

Hunter, A. (1998). Possum and the peeper. Boston: Houghton Mifflin. 
Justice, L. M., Meier, J., \& Walpole, S. (2005). Learning new words from storybooks: An efficacy study with at-risk kindergartners. Language, Speech, and Hearing Services in Schools, 36(1), 17-32.

Lawrence, M. (2000). The caterpillar that roared. New York: Dorling Kindersley.

Lionni, L. (1963). Swimmy. New York: Alfred A. Knopf.

Marulis, L. M., \& Neuman, S. B. (2010). The effects of vocabulary intervention on young children's word learning: A meta-analysis. Review of Educational Research, 80(3), 300-335.

Mayer, M. (1973). What do you do with a kangaroo? New York: Scholastic.

Maynard, K. L., Pullen, P. C., \& Coyne, M. D. (2010) Teaching vocabulary to first-grade students through repeated shared storybook reading: A comparison of rich and basic instruction to incidental exposure. Literacy Research and Instruction, 49(3), 209-242.

Pile, E. J. S., Girolametto, L., Johnson, C. J., Chen, X., \& Cleave, P. J. (2010). Shared book reading intervention for children with language impairment: Using parents-as-aides in language intervention. Canadian Journal of Speech-Language Pathology and Audiology, 34(2), 96-109.

Rice, M. L., Oetting, J. B., Marquis, J., Bode, J., \& Pae, S. (1994). Frequency of input effects on word comprehension of children with specific language impairment. Journal of Speech \& Hearing Research, 37(1), 106-121.

Roskos, K., Ergul, C., Burstein, K., Christie, J., \& Han, M. (2008). Who's learning what words and how fast? Preschooler's vocabulary growth in an early literacy program. Journal of Research in Childhood Education. 22(3). 275-290. 
Small, D. (1985). Imogene's antlers. New York: Crown.

Wells, R. (1988). Shy Charles. New York: Penguin Books.

\section{Acknowledgements}

The data described here are from an ongoing clinical trial supported by Grant DC 012824 from the National Institutes of Health (NIH). The contents are solely the responsibility of the authors and do not necessarily represent the official views of the NIH. We thank the children and their families who participated in this research. We also thank the SpeechLanguage Pathologists, the school directors and the teachers for their involvement and support. Finally, we thank the staff of the KAW Story Project for their assistance with participant recruitment, data collection, and data processing. 


\section{Learning Outcome}

Learners will be able to describe the components of a high intensity interactive book reading treatment for kindergarten children with SLI.

1. The most promising intensity of interactive book reading for children with SLI is:
a. 12 exposures
b. 24 exposures
c. $36 \underline{\text { exposures }}$
d. 48 exposures

Answer: C, As noted in the article, "current results suggest that 36 exposures is the most promising intensity for children with SLI."

2. Tier two words are:
a. Very low frequency words for mature language users
b. Low to medium frequency words for mature language users
c. Medium to high frequency words for mature language users
d. Very high frequency words for mature language users

Answer: C, As noted in the article, tier two words are medium- to high-frequency words for mature language users.

3. How many exposures to-do children receive to the target words during a treatment session?
a. 4
b. 6
c. 8
d. 10

Answer: B, Children receive 6 exposures as illustrated in Figure 1

4. To achieve 36 total exposures to each treated word, the books are read:
a. 3 times
b. 4 times
c. 5 times
d. 6 times

Answer: D, Books are read 6 times as illustrated in Figure 4.

5. Interactive book reading can be used with children with SLI to
a. Build vocabulary
b. Expand phonetic repertoire
c. Improve grammar
d. Enhance pragmatics

Answer: A, The article emphasizes that interactive book reading can improve vocabulary. 


\section{Page 17 of 20}

1

2

3

4

5

6

7

8

9

10

11

12

13

14

15

16

17

18

19

20

21

22

23

24

25

26

27

28

29

30

31

32

33

34

35

36

37

38

39

40

41

42

43

44

45

46

47

48

49

50

51

52

53

54

55

56

57

58

59

60

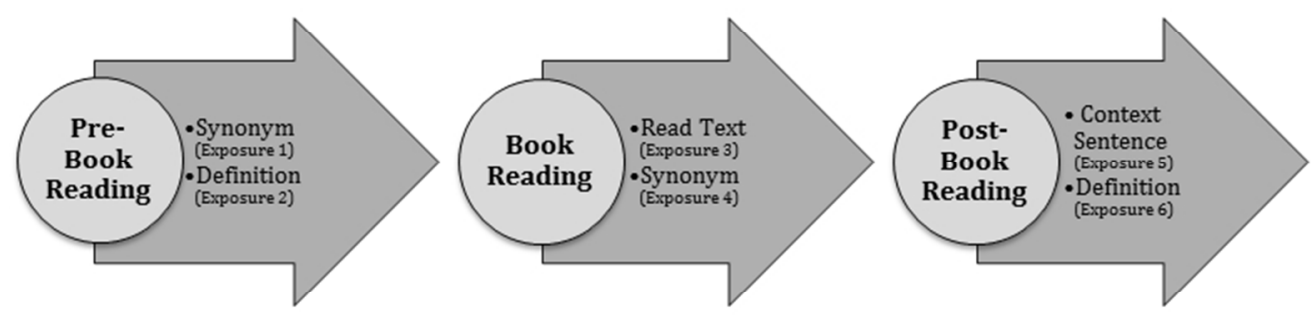

Figure 1. This is the order of an individual treatment session. This figure depicts the protocol for each individual book. The child will be exposed to each target word a total of 6 times. $206 \times 82 \mathrm{~mm}(96 \times 96 \mathrm{DPI})$ 
Pre-book reading stimulus card. Photo taken by Bruce Kerridge, Sydney, Australia. $120 \times 88 \mathrm{~mm}(96 \times 96 \mathrm{DPI})$ 


\section{Page 19 of 20}

1

2

3

4

5

6

7

8

9

10

11

12

13

14

15

16

17

18

19

20

21

22

23

24

25

26

27

28

29

30

31

32

33

34

35

36

37

38

39

40

41

42

43

44

45

46

47

48

49

50

51

52

53

54

55

56

57

58

59

60

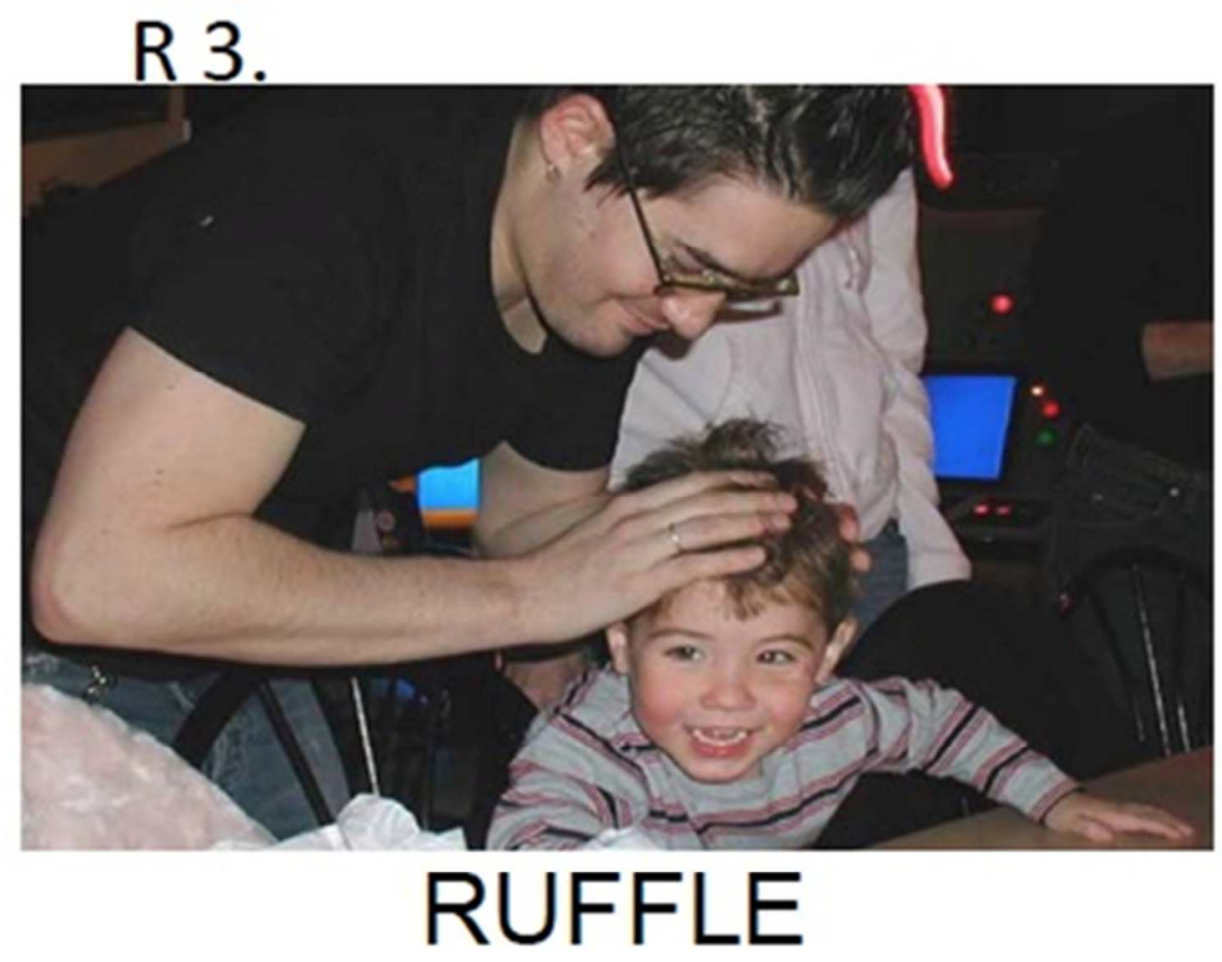

Post-book reading stimulus card. Photo downloaded from http://duanerussell.com/Jordan/index.htm. $147 \times 112 \mathrm{~mm}(96 \times 96 \mathrm{DPI})$ 
Figure 4 Visual Schedule for Intensity 36. Each book was read 6 times (see number in parentheses) and the $99 \times 156 \mathrm{~mm}(96 \times 96 \mathrm{DPI})$

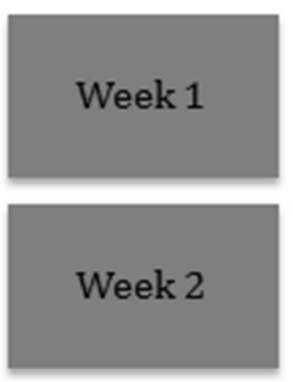

Tx Session 1
-Book 1 (1st)
-Book 2 (1st)

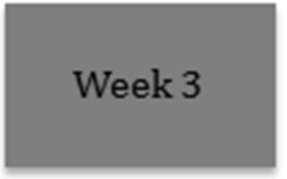

\section{Tx Session 3}

- Book 5 (1st)

- Book1 (2nd)

\section{Tx Session 5}

- Book 3 (2nd)

-Book 5 (2nd)

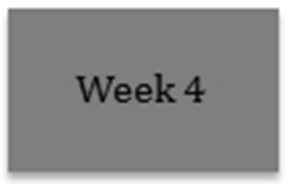

\section{Tx Session 7}

-Book 2 (3rd)

- Book 5 (3rd)

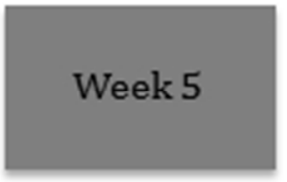

Tx Session 9

-Book 3 (4th)

-Book 2 (4th)

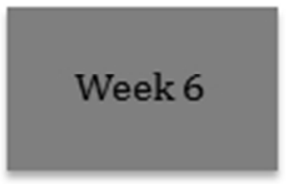

Tx Session 11

-Book 2 (5th)

-Book 1 (5th)

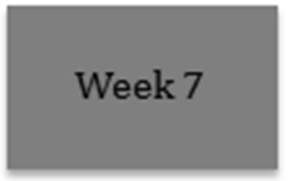

Tx Session 13

-Book 1 (6th)

-Book 5 (5th)

\section{Tx Session 2 \\ -Book 3 (1st) \\ -Book 4 (1st) \\ Tx Session 4 \\ -Book 2 (2nd) \\ -Book 4 (2nd)}

\section{Tx Session 6 \\ -Book 1 (3rd) \\ -Book 4 (3rd)}

\section{Tx Session 8}

- Book 3 (3rd)

-Book 1 (4th)

\section{Tx Session 10}

-Book 5 (4th)

-Book 4 (4th)

Tx Session 12

-Book 4 (5th)

- Book 3 (5th)

\section{Tx Session 14 \\ -Book 4 (6th) \\ -Book 2 (6th)}

\section{Week 8}

Tx Session 15

-Book 5 (6th)

-Book 3 (6th)

\section{order of the books was rotated across sessions.}

